\title{
Experiences of a chief registrar - a report from the Nottingham University Hospitals chief registrar
}

\author{
Author: Francis Kynaston-Pearson*
}

\section{Introduction}

The chief registrar (CR) programme seeks to establish a new senior leadership role for trainee doctors. The current Nottingham University Hospitals (NUH) CR has focused on morale building by improving trainee doctors' experience and engagement with the trust. An enhanced sense of belonging may help alleviate the alienation some doctors experience from the nomadic existence their rotational placements can create.

\section{Methods}

Three areas of the quality improvement project (QIP) have been a particular focus: (i) making the best first impression; (ii) facilitating transition and rotation; and (iii) celebrating success.

\section{Making the best first impression}

NUH induction feedback was suboptimal and included reports that multiple emails received from different sources prior to starting created confusion. The CR worked with a task and finish group seeking to address this among other issues. A single welcome letter and new universal handbook are among some of the outcomes and the CR is leading a pan-trust QIP reviewing and improving the process of local departmental induction.

Unlike the process of clinical handovers, where some guidance exists, no such guidance exists for ward-based handover despite evidence that, for example, induction handbooks' improve doctors' confidence and efficiency. The QIP has completed local induction guides on the pilot wards with handover videos called 'Doctors' voices' being produced for the second cycle of improvement.

\section{Facilitating transition and rotation}

While it is not surprising some wards at NUH stock highly specialist equipment relevant to their clinical area, it is surprising to find no uniform layout to notes trolleys and basic equipment drawers, with some wards sometimes lacking basic equipment items. This can result in doctors wasting precious minutes on routine tasks searching for such items or indeed present potential safety concerns. Taking inspiration from 'The productive ward', another QIP compared a survey of doctors' responses in what they considered vital equipment to the current provisions. The authors propose a new doctors on-call backpack while they cover multiple wards and draws on the concept of a standardised trolley to improve time efficiency.

\section{Celebrating success}

While induction can create a good impression, NUH (like many other trusts) lacks an equivalent send-off. Despite many trainees undertaking and presenting projects from NUH at national and international conferences, much of this work is not readily publicised within the trust. While NUH benefits from a successful staff awards scheme each November ('NUH honours'), trainees who rotate in August often fail to gain a nomination. The CR has secured funding and a venue for the first 'NUH doctors in training' conference providing a platform for established academic trainees to inspire others and the perfect stepping stone for junior trainees to showcase smaller projects. There will be inspirational workshops from the NUH talent pool and clinical skills lab training in hard to access procedures (eg chest drains). The event will close with the 'Postgraduate Medical Education Awards' ceremony recognising and celebrating outstanding contributions by certain trainees.

\section{Results}

The results and discussion of the above projects were presented at the Medicine 2019 conference.

\section{References}

1 National Health Service Institute for Innovation and Improvement. The Productive Ward: Releasing time to care. NHSI, 2009.

Author: Nottingham University Hospitals NHS Trust ${ }^{*} \mathrm{RCP}$ chief registrar 